\title{
Electronic stethoscope - a didactic and low cost acquisition system for auscultation
}

\begin{abstract}
Background: This paper presents an electronic stethoscope model for cardiac auscultation, that adds innovative functionality to the conventional stethoscope, nominated "Electronic Stethoscope". Among the Electronic Stethoscope functionalities, can be cited the volume adjust in order to facilitate the hearing of the heart pulses, the graphic presentation of the heart beat sound waveforms, from a monitor, and the store of the cardiac auscultation data in a memory card for future analysis. It is believed that this additional functions, nonexistent in conventional stethoscopes, can contribute in the identification of pathology anomalies by the doctors and medicine students, growing the diagnoses trusting rate. Beyond that, the stored data in the equipment can be visualized and listened, without the necessity of the patient presence, characteristic that enables the build of a database, for example, of indicative signals of pathologies that could be used in class and practicing. With this equipment it is also possible to make adjusts on amplitude and length of the graphics (zoom), for a better details visualization. Undesirable ambient sounds can also be mitigated by the use of low-pass digital filters of IIR type implemented on the stethoscope software. This equipment has also a graphic resource for the identification of patient's heart rate similar of the gestational ultrasound equipment. Results of interviews with doctors and medicine students shows that the equipment have practical applicability, either in clinic or classroom, and extremely intuitive mode of operation, which would require no prior specific training.
\end{abstract}

Keywords: electronic stethoscope, auscultation analysis, heart rate
Volume 5 Issue 5 - 2019

Felipe Silveira Marques Lisboa, José Saad Said

Neto,Veronica Isabela Quandt, Leonardo Gomes Tavares

Department of Electrical Engineering, Universidade Positivo, Brazil

Correspondence: Veronica Isabela Quandt, Department of Electrical Engineering, Universidade Positivo, R. Professor Pedro Viriato Parigot de Souza, 5300, Campo Comprido, Curitiba, PR, Brazil,Tel +55 4 I 3526-5I53, Email veronica.quandt@up.edu.br

Received: October 06, 2019 | Published: October 15, 2019

\section{Introduction}

Auscultation is a method of physical examination that aims to listen to the internal sounds of the body, and is made since the 4th century BC that consisted of placing the ear directly on the skin of the sick to hear the sounds of the organs. ${ }^{1}$ In 1816, French physician René Laennec invented the first stethoscope, which consisted of simple tubular equipment capable of capturing and conducting cardiac and vascular vibrations from the thoracic surface to the hearing aid. In addition, the stethoscope eliminates some noises present in the environment where the consultation is made. Over time the stethoscope has been increasingly developed, with design changes for better sensing. ${ }^{2}$ Electronic stethoscopes are currently being commercially developed and are becoming quite popular. They utilize digital signal processing technology with wireless transfer to the computer, entering the digital world. ${ }^{2,3}$ SoundScope is an electronic stethoscope for healthcare professionals, especially doctors. Its main features are: amplification and storage of auscultated sounds, and patient data management. It was developed by students from the Federal University of Paraná in mid-2013. ${ }^{4}$ There are similar equipment on the market, but with different functionalities. Some do audio storage, others do volume amplification. A widely used device is the Jabes Digital Analyzer Stethoscope. With it you can do cardiac auscultation, amplify the volume and make the recording so that you can visualize graphically with a program developed by the company itself. ${ }^{5}$

Huang et al. developed a portable multifunctional electronic stethoscope. ${ }^{6}$ It is capable of listening to and transferring the signal via bluetooth to a smartphone. It consists of a data acquisition module, a processing module, a transmission module and a storage module. The system shows the signal acquired in time and frequency. Yilmaz et al. ${ }^{7}$ designed and developed a low-cost, easy-to-wear electronic stethoscope that is easy to integrate into a garment. ${ }^{7}$ They then analyzed the noise and linearity of the recorded sound signal and compared the prototype with a standard stethoscope, widely used in clinical practice. These studies show that the subject can be widely explored and with different proposals. Regardless of the development and innovation of the stethoscope, a heart auscultation requires a good understanding of how sounds and their characteristics are. Since medical students and doctors have difficulty identifying pathologies, because the theoretical method of teaching is not sufficient for learning and, throughout the medical course, there is not enough experimentation with the auscultation exam. ${ }^{8}$ In research with doctors and medical students during this project, it was reported that there is some difficulty in identifying murmurs, arrhythmias and other types of heart problems. In the research, many medical students said there were differences of opinion regarding cases of cardiac auscultation, where some said to hear murmur and others not. In this case, the electronic stethoscope would help as an aid for medical students to identify and improve learning. The doctors, on the other hand, said that it was difficult to hear murmurs, because they are very difficult to hear, for pathological reasons or because there is a fat layer between the stethoscope and the heart. In this case, the doctor pointed out as a possible aid the graphic interface and the possibility of increasing the signal volume in real time. Incorrect diagnoses in clinical examinations can cause various setbacks, making it difficult to cure pathologies later and also 
an additional expense of hospital and patient resources. In order to reduce these difficulties, the purpose of an electronic stethoscope is to bring something more complete, low cost and innovative. The proposed equipment will provide medical assistance and a better prediagnosis to the patient, enabling them to have a sense of sight aid together with hearing, enabling students to have a visual notion of sound, facilitating the identification of pathologies. It also promotes greater agility at the time of the exam and helps to make a more accurate diagnosis.

Regarding the sounds and murmurs, with the proposed electronic stethoscope, it may be feasible to jointly analyze several characteristics at the same time, such as intensity, frequency and timbre. Knowing that the characteristics are present in the sound signal itself, it is also important to identify the position of the blows on the noises due to the graphic form that will be presented to the hearing aid. The sound tone, for example, can be better identified in the equipment, being able to observe its frequency spectrum. In addition, the proposed electronic stethoscope may allow the storage of these auscultatory signals, enabling the construction of a database in order to facilitate future research and comparison of sound signals. Thus, the proposed work was to develop a prototype of a device capable of assisting doctors in cardiac diagnostics, so that the volume of cardiac auscultatory sounds can be adjusted and graphical recorded. Besides being functional, this prototype must be of low cost, precisely in order to be used by medical students in didactic form.

\section{Methods}

For the development of the electronic stethoscope, the project was divided into two parts, hardware and software. The idea was to create a product that is capable of assisting physicians in cardiac auscultation by enlarging the sound wave graph of the heartbeat, increasing the auscultation volume and storing this data on an SD card for future analysis. The system, according to Figure 1, consists of 2 modules, which are the Sensor and Microcontroller, forming, together with the peripherals, the HMI module (human machine interface). - Sensor: Consists of a component transducer (electret microphone) that transforms a mechanical signal into electrical, diaphragm and bell, where the mechanical signal is amplified:

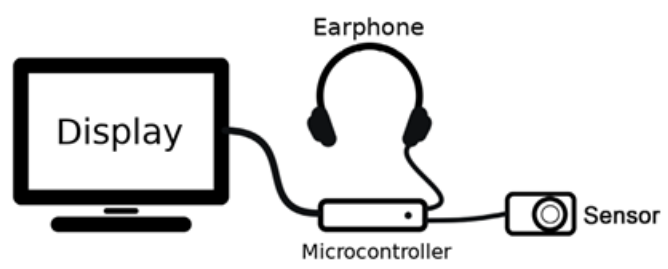

Figure I Project Schematic.

I. Microcontroller: The part of the system where all signal processing happens as well as the graphical application through a microcontroller.

II. HMI: Module where the user interaction with the system will occur, containing the created software, the monitor for the graphical interface display and headphones, these last two peripheral equipment being non-part of the work.

\section{Scaling and calculation memorial}

Aiming at a system that allows the application of filters to better analyze the audio signals, second order Butterworth and low pass type IIR filters were designed. The cut-off frequencies were 200, 500, 800 and $1000 \mathrm{~Hz}$ for the sampling frequencies of $16000 \mathrm{~Hz}$ and $44100 \mathrm{~Hz}$, and the coefficients of these filters can be seen in Table 1 and Table 2, respectively, for the graphical part, and the playback part of the audio, in real time. Butterworth 200,500, 800 and $1000 \mathrm{~Hz}$ low pass filters have been designed through an online interface called octave-online. ${ }^{8}$ To design the filter through the site, it is necessary to say the sampling frequency of the signal and the cut-off frequency of the filter to be designed. Example: $\mathrm{Fs}=44100 \mathrm{~Hz}$ and $\mathrm{Fc}=500 \mathrm{~Hz}$. Next, the "butter" class was used to make the second-order butter worth filter. Finishing with the input of the filter data, the program returns the coefficients of the poles (An) and zeros (Bn) completing the IIR filter design.

Table I Sampling frequency: $16000 \mathrm{~Hz}$

\begin{tabular}{llllll}
\hline $\begin{array}{l}\text { Cut-off } \\
\text { frequency } \\
(\mathbf{H z})\end{array}$ & b0 & bl & b2 & al & a2 \\
\hline 200 & 0.0014603 & 0.0029206 & 0.0014603 & -1.88903 & 0.98487 \\
500 & 0.0084427 & 0.0168854 & 0.0084427 & -1.72378 & 0.75755 \\
800 & 0.020083 & 0.040167 & 0.020083 & -1.56102 & 0.64135 \\
1000 & 0.029955 & 0.059909 & 0.029955 & -1.45424 & 0.57406 \\
\hline
\end{tabular}

Table 2 Sampling frequency: $44100 \mathrm{~Hz}$

\begin{tabular}{llllll|}
$\begin{array}{l}\text { Cut-off } \\
\text { frequency } \\
(\mathbf{H z})\end{array}$ & b0 & bl & b2 & al & a2 \\
\hline 200 & $1.9897 \mathrm{e}-4$ & $3.9794 \mathrm{e}-4$ & $1.9897 \mathrm{e}-4$ & -1.95971 & 0.96050 \\
500 & 0.0012074 & 0.0024148 & 0.0012074 & -1.89933 & 0.90416 \\
800 & 0.0030029 & 0.0060058 & 0.0030029 & -1.83912 & 0.85113 \\
1000 & 0.0046040 & 0.0092080 & 0.0046040 & -1.79910 & 0.81751 \\
\hline
\end{tabular}

\section{The overall design of the hardware}

The hardware used was the Orange Pi Zero Plus 2, due to its pertinent characteristics necessary for the project, the most important being HDMI and audio input and output, as well as good processing capacity, SD memory card and connection via Wi-Fi. It was used in conjunction with the Orange $\mathrm{Pi}$, an extension card, to be able to use audio output and USBs using connectors as well as the microphone. For the use of a graphical application, a robust Operating System was chosen, capable of performing all the tasks that were necessary for the development of the Software, thus a Debian distribution Linux image, Stretch version was used, which showed the best performance for the microcontroller. In addition, for storage of the Operating System and other project files, such as the program itself, audios and source code, a 16 GB SD card was used. Other equipment choices for the prototype to function properly were the AC/DC $5 \mathrm{~V} 3 \mathrm{~A}$ power supply required by the card and an HDMI / VGA adapter as well as the VGA connector for connection to the display. In the development of the project, the original microphone was removed from the board, as there was a need to add a cable so that it could place the diaphragm with the electret microphone in the region where the cardiac auscultation is 
made. Listening to cardiac sounds with the electret microphone alone could not capture characteristic heart sounds, so it was necessary to use the conventional stethoscope diaphragm to amplify them, thus being noticeable by the transducer. First, an electret microphone with a diameter larger than the conventional stethoscope diaphragm connection was used, which was later replaced by an electret microphone with a smaller diameter.

\section{The overall design of the software}

For the development of the system the platform QT 5.7.1 was used for human machine interface, where it was possible to perform the programming in $\mathrm{C}++$, code adaptations and libraries. In conjunction with the hardware equipment chosen, software was developed with a QT interface capable of sampling the waveforms of the original and filtered heartbeats, as well as allowing them to be adjusted and initiating other features such as audio recording and measurement of the heartbeat rate. With the need for better quality and to capture all frequencies that the human ear can pick up, filter out noise and sample the signal, the following parameters were used:

a) Sampling Frequency (Audio): $44100 \mathrm{~Hz}$

b) Sampling Frequency (Graph): $16000 \mathrm{~Hz}$

c) Sample size: 16 bits

d) Cut-off frequency for the designed IIR filters: 200, 500, 800 and $1000 \mathrm{~Hz}$

e) Data processing buffer: 4096 samples

f) Chart Width (zoom): Up to $5 x$

g) Number of samples: 100-12000 samples

The software has been programmed to automatically perform all calculations and hardware elements connection with the application, as well as audio capture, recording and signal filtering. The method used to measure heartbeat rate was to verify the period between each cardiac cycle, and then to find out the frequency of the signal. A function was created that, shortly after pausing the real-time graph, located the coordinates, in pixels, by a double mouse click event and later converted to cartesian coordinates for the signal samples. Thus it was possible to find the $\mathrm{x}$ axis coordinate, referring to the sample position. The same process being performed for the second point of the plan. Another function was created to find the distance between these two samples located on the $\mathrm{x}$ axis, and to paint a longitudinal line on the graph so that the location of the two selected coordinates could be visually perceived, as well as the distance between them. Knowing the sampling frequency, the distance of the samples can be converted into a unit of time, thus discovering the signal period, and then the frequency of the signal, given in beats per minute.

In the interface developed for graph visualization, there are functions for better signal visualization, through dials for graph amplitude and length, buttons for start, record, pause, guide change and volume. The commands for graph adjustments that can increase the amplitude up to $5 \mathrm{x}$ and the length of $100-12000$ samples are referenced in items (9 and 11) of Figure 2, respectively. Buttons (3 and 4) are for real-time start and stop listening, while buttons (5 and 6) are for start and stop recording, in which case, for a better view of the user, a text was arranged in the graph pointing the recording time. Filter options (7), volume adjustments (8), tab switching (12) and file opening have already been saved (2). Being the status of the commands shown in the message bar (13). In addition, it is possible to measure the heartbeat rate by double clicking on the graph to mark the beginning and end of a signal period, which after selection, the heartbeat is shown in item (1) of Figure 2.

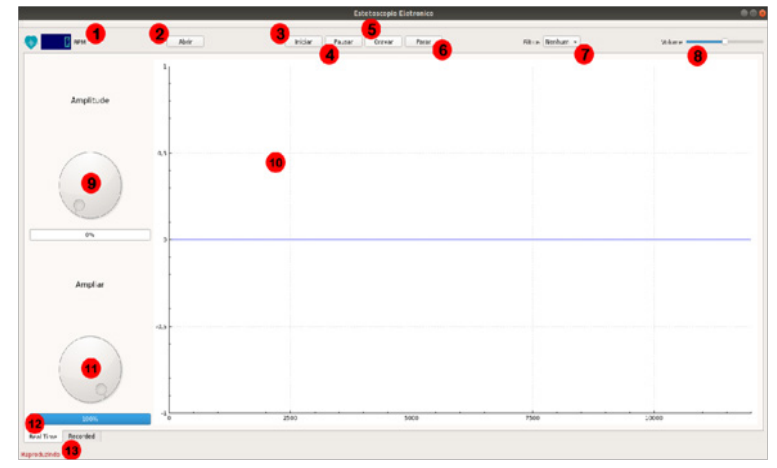

Figure $\mathbf{2} \mathrm{SW}$ interface.

\section{Results}

After cost-effective project studies, the prototype shown in Figure 3 was performed. In the prototype the microprocessor was protected by a case with proper holes for the audio, USBs and HDMI inputs, from which comes a cable that connects to the microphone and sensor used, surrounded by a protection that leaves only the diaphragm, which will be used for auscultatory analysis, the bell being hidden by the protection. A foam case was also assembled for the storage and protection of the prototype as a whole, and a design logo was added for a better presentation of the model. The cost of assembling the prototype was around USD 120. Considered a low cost when looking at the price of a commercial digital stethoscope, which costs around USD $400 .^{10}$

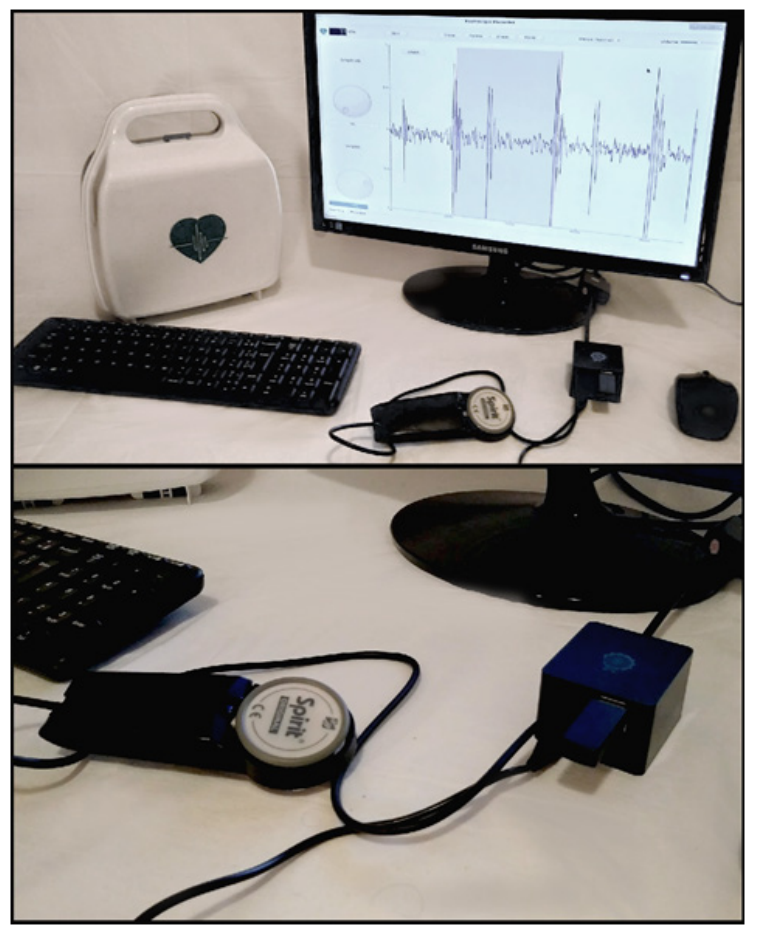

Figure 3 Electronic stethoscope prototipe. 


\section{Functionality test}

With the software it was possible to visualize and to hear the characteristic sounds of the heart, being able to also record them in an SD card. This is due to all the programming involved, where filters were designed to eliminate noise and the ability to modify the amplitude and length of the graph. By auscultation of the heart, it was possible to obtain the signals of the heartbeat being captured, the first sound and the second sound respectively, both without any software filtering, as shown in Figure 4. It can be observed in the cardiac auscultation that the sampling of the graph was satisfactory, leaving the samples clearly visible. Knowing that the frequency of heart sounds ranges from 20 to $500 \mathrm{~Hz}$, it has been found that the $500 \mathrm{~Hz}$ filter is ideal for a cardiac cycle analysis, as shown in Figure 5 For the measurement of the heartbeat, the method of identifying the period of the captured signal was used, where in Figure 6 the limits are shown in the graph and in the upper left corner the heartbeat of the signal. The use of the prototype was compared with a heartbeat rate measuring device so that the quality and robustness of the measurement could be verified. After testing, the system proved to be accurate and faster because with just two clicks the heart rate is sampled on the display.

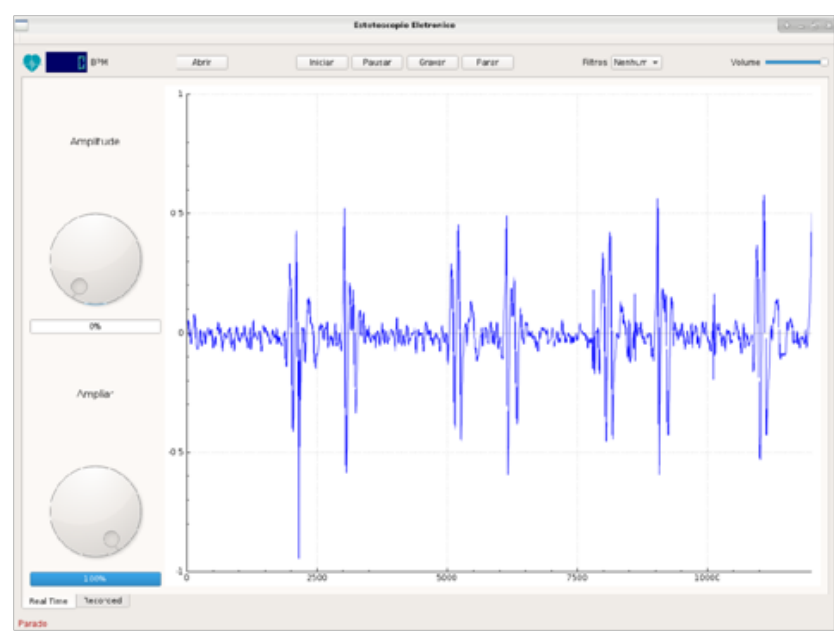

Figure 4 SW signal displayed.

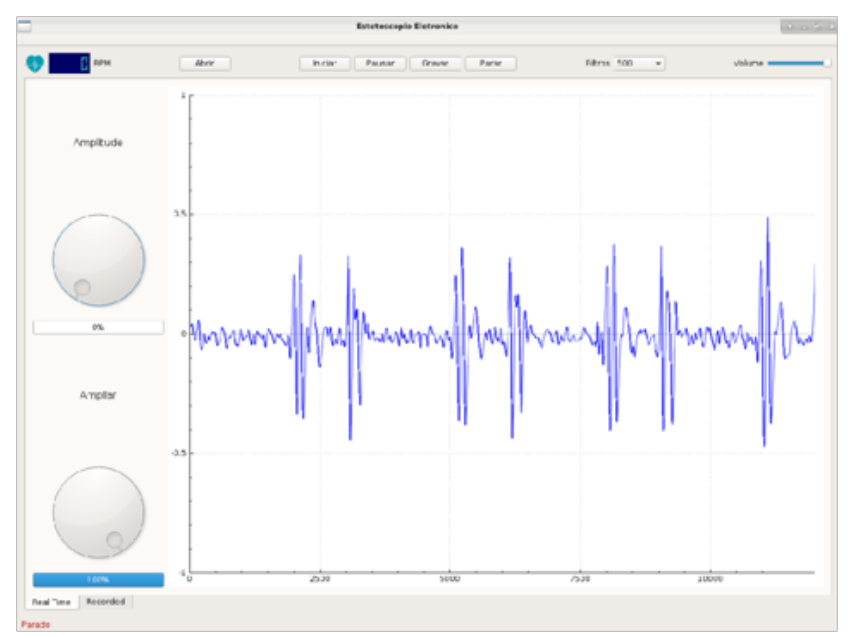

Figure 5 Filtered signal $(\mathrm{Fc}=500 \mathrm{~Hz})$.

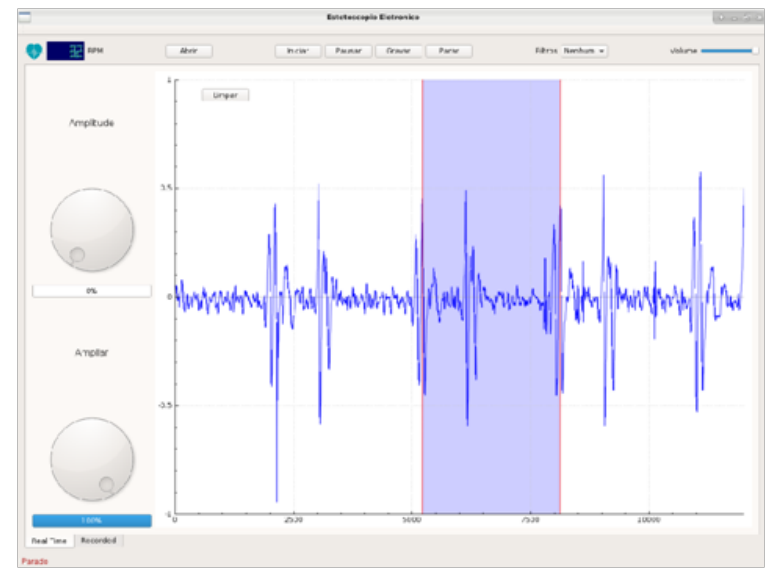

Figure 6 BPM measurement.

\section{Quantitative test}

In seeking the applicability of the project, questions were asked to doctors and medical students, seeking to improve the project. Overall, the project was positively qualified, claiming to be an interesting equipment, where the aid of graphics could facilitate the identification of murmurs and arrhythmias in general. One of the applications would be the use for auscultation of murmurs in newborns, as it is easy to identify and would help academics. Regarding the designed filters, they would be interesting in hospital environments where there is a lot of movement, such as the emergency room or traffic routes, where the doctor needs to do pre hospital care. Another point mentioned was the use of the equipment by nurses so that it can expedite the patient screening process as needed. Suggestions were given to improve the system as well, among them making the equipment more practical, being able to adapt to the mobile phone, improving the audio quality and also being able to amplify the volume even more.

\section{Discussion}

The Electronic Stethoscope developed as a graduation work of electrical engineering students, presented some advantages and disadvantages compared to the equipment mentioned in the introduction. The electronic stethoscope has some advantages over SoundScope, because with the electronic stethoscope it is possible to view the graph of the heartbeat in real time, whereas with SoundScope, it is possible only with recorded sounds. Another positive point is the interface developed in the electronic stethoscope, which is selfcreated and easy to handle. Compared with the Jabes Digital Analyzer Stethoscope, the electronic device maintained its positive point over real-time graphical visualization of cardiac auscultation, but Jabes maintained superiority over audio quality, amplification and comfort. In the future, the Electronic Stethoscope can be enhanced with machine learning, to provide another tool to aid the doctor. Machine learning works as follows, during the patient's cardiac auscultation, the algorithm reads it and then compares it (using artificial intelligence algorithms) with the machine's database graphs. Through these comparisons, the machine may suggest pathologies that patients may have. As for the prototype, possible improvements would be in relation to the visual finish of the system, and a 3D protection could be developed for the use of the two sensors, bell and diaphragm, because 
with this model it is not possible to mechanically change the function of the conventional stethoscope.

\section{Conclusion}

Based on the project performed and the results obtained, it can be concluded that the electronic stethoscope can be a device of use to aid in cardiac auscultation. Because the equipment is a tool that relates the sense of sight and hearing, where you can observe the sound behavior of the heartbeat, S1 and S2 signals. With an electronic stethoscope you can plot the sound wave of the heartbeat, make changes in the amplitude and length of the graph, increase the volume, calculate the heartbeat per minute, record the data obtained on an SD card for future analysis, and add filters to soften ambient noise. It can also be concluded that it can be built with low cost and is intended for didactic use in medical, nursing and other health-related courses.

\section{Funding details}

None.

\section{Acknowledgments}

None.

\section{Conflicts of interest}

Authors declare that there is no conflict of interest.

\section{References}

1. Ferraz AP, Soares BS, Terra DA, et al. The history of stethoscope and cardiac auscultation. Revista Médica de Minas Gerais. 2011;21(4):479-485.

2. Permin H, Norn S. Stethoscope-Over 200 years. 2019.

3. Leng S, San Tan R, Chai KT, et al. The electronic stethoscope. Biomedical engineering online. 2015;14(1):66.

4. Santos FG, Tambosi GP. Estetoscópio eletrônico microcontrolado. Universidade Tecnológica Federal do Paraná; 2013.

5. JABES Life Sound System. 2019.

6. Huang H, Yang D, Yang X, et al. Portable multifunctional electronic stethoscope. In2019 IEEE 3rd Information Technology, Networking, Electronic and Automation Control Conference (ITNEC). 2019:691-694.

7. Yilmaz G, Starkov P, Crettaz M, et al. A Low-Cost USB-Compatible Electronic Stethoscope Unit for Multi-channel Lung Sound Acquisition. InMediterranean Conference on Medical and Biological Engineering and Computing. 2019:1299-1303.

8. Mesquita CT, Reis JC, Simões LS, et al. Digital stethoscope as an innovative tool on the teaching of auscultatory skills. Arquivos brasileiros de cardiologia. 2013;100(2):187-189.

9. OctaveOnline. 2019

10. allheart. 2019 . 